\title{
A femtosecond fluorescence study of vibrational relaxation and cooling dynamics of UV dyes $\dagger$
}

\author{
Olivier Braem, ${ }^{a}$ Thomas J. Penfold, ${ }^{a b c}$ Andrea Cannizzo ${ }^{a d}$ and Majed Chergui ${ }^{a}$ \\ Received 6th October 2011, Accepted 16th January 2012 \\ DOI: $10.1039 / \mathrm{c} 2 \mathrm{cp} 23167 \mathrm{k}$ \\ We present a femtosecond broad-band fluorescence up-conversion study of the vibrational \\ relaxation dynamics of two UV chromophores, 2,5-diphenyloxazole (PPO) and para-terphenyl \\ $(p \mathrm{TP})$, pumped with a large excess of vibrational energy $\left(>2000 \mathrm{~cm}^{-1}\right)$. The band narrowing of \\ the transient fluorescence spectrum reflects a biphasic cooling process in a few hundreds of fs and \\ a few ps. In the sub-ps regime, our data suggest a structural rearrangement in the excited state, \\ followed by thermalization of the excess energy. These dynamics affect the fluorescence spectra of \\ PPO and $p$ TP in different ways. In PPO, the damping of a low frequency vibrational wavepacket \\ and a significant sub-ps narrowing of the band characterize the vibrational relaxation. In $p \mathrm{TP}$, the \\ latter is faster and appears as a red shift with distortion of the band in $<200 \mathrm{fs}$.
}

\section{Introduction}

Intramolecular vibrational redistribution (IVR) and vibrational energy transfer (VET) are known to play crucial roles in a large range of photochemical reactions such as charge transfer, ${ }^{1}$ isomerization ${ }^{2-5}$ or even protein dynamics. ${ }^{6,7}$ Accordingly, the timescales and the pathways of vibrational energy relaxation are important issues in condensed phase photochemistry. Over the past decades, the study of such processes has been developed thanks to ultrafast spectroscopic techniques such as transient absorption spectroscopy. ${ }^{8-11}$ While femtosecond-resolved fluorescence has been more widely used to probe solvation dynamics, i.e. to characterize the solvent response to a photo-induced dipole change in a solute chromophore, ${ }^{12-16}$ this approach has also been used, to a lesser extent, to study vibrational relaxation. ${ }^{17-19}$ The dyes that were used to investigate such processes absorb in the

\footnotetext{
${ }^{a}$ Laboratory of ultrafast Spectroscopy, ISIC, Ecole Polytechnique Fédérale de Lausanne, Switzerland $\mathrm{CH}-1015$.

E-mail: majed.chergui@epfl.ch; Fax: +41(0)216930365;

Tel: $+41(0) 216930447$

${ }^{b}$ Paul-Scherrer Institut, Swiss FEL, CH-5232 Villigen, Switzerland

${ }^{c}$ Laboratory of computational chemistry and biochemistry, ISIC,

Ecole Polytechnique Fédérale de Lausanne, CH-1015, Switzerland

${ }^{d}$ Current address: Institute of Applied Physics, University of Bern,

Silderstr. 5, CH-3012 Bern, Switzerland

$\dagger$ Electronic supplementary information (ESI) available: contains (i) figures of $\left(M_{0}(t)\right)$ of the dyes fluorescence; (ii) figures of the transient fluorescence spectra of the dyes with their F-C fits; (iii) Fourier Transform spectra of the oscillations pattern of $M_{1}(t)$; (iv) the temperature dependence of the steady state fluorescence spectra; (v) the zero-phonon-line and width obtained from the $\mathrm{F}-\mathrm{C}$ fit; (vi) a figure of the eigenvectors and frequencies of the first 8 calculated low frequency normal modes of the PPO molecule; (vii) a table of the calculated vibration frequencies of 2,5-diphenyloxazole and para-terphenyl. See DOI: $10.1039 / \mathrm{c} 2 \mathrm{cp} 23167 \mathrm{k}$
}

visible region and are large and complex molecules. Smaller and simpler dye molecules are more appropriate for detailed theoretical and experimental characterizations, but they usually absorb and emit in the UV region. The extension of the fluorescence up-conversion technique with broadband detection to the UV has demonstrated promising capabilities to follow vibrational relaxation in real time. ${ }^{12,20}$ Here, we focus on the early dynamics of UV chromophores, from the end of the ultrafast intramolecular vibrational redistribution (IVR) to the complete transfer of the excess energy (heat) to the bulk solvent.

For this purpose, we investigated the femtosecond-resolved fluorescence of two different UV dyes, namely 2,5-diphenyloxazole ( $\mathrm{PPO}$ ) and para-terphenyl $(p \mathrm{TP})$, whose photophysical properties are summarized in Table 1. Their absorption and emission spectra are plotted in Fig. 1. The high photostability, emission quantum yield and large Stokes shift of PPO have led to its extensive use as a wavelength shifter in liquid scintillators, as well as a laser dye. ${ }^{21-26}$ The first absorption band peaking around $310 \mathrm{~nm}$, assigned to the $\mathrm{S}_{1} \leftarrow \mathrm{S}_{0}$ transition, shows a vibronic structure in non-polar solvents, which exhibits at least two progressions. The structure becomes blurred in polar solvents (Fig. 1). The fluorescence in non-polar solvents exhibits a near mirror image with respect to the absorption band, and a well resolved vibrational structure with an apparent average spacing of $1370 \mathrm{~cm}^{-1}, 28$ arising from the coupling with two modes at $1544 \mathrm{~cm}^{-1}$ and $980 \mathrm{~cm}^{-1}$ assigned to oxazole ring $\mathrm{C}=\mathrm{N}+\mathrm{C}=\mathrm{C}$ stretching and deformation modes. ${ }^{31-33}$ In cyclohexane, the absorption and fluorescence spectra clearly deviate from the mirror image symmetry, suggesting a structural change in the excited state.

$p$ TP has been extensively used as a crystalline environment for single molecule spectroscopy measurements, ${ }^{34-36}$ and was studied 
Table 1 Photophysical properties of three studied UV dyes in methanol and cyclohexane

\begin{tabular}{llllllll}
\hline Compound & $\tau_{\text {fluo }}{ }^{a} / \mathrm{ns}^{27}$ & $\lambda_{\text {abs }}{ }^{b} / \mathrm{nm}$ & $\lambda_{\mathrm{em}}{ }^{c} / \mathrm{nm}$ & Q.Y. $^{d}$ & Radiative transition & Ground state dipole & Excited state dipole \\
\hline 2,5-Diphenyloxazole & & & & & & & \\
Methanol & 1.65 & 303 & 362 & $0.5^{28}$ & $\mathrm{~S}_{1} \rightarrow \mathrm{S}_{0}$ & $1.59^{29}$ & \\
Cyclohexane & 1.36 & 303 & 357 & $0.85^{28}$ & & & \\
$\begin{array}{l}\text { Para-terphenyl } \\
\text { Methanol }\end{array}$ & 1.17 & 274 & 339 & $0.93^{30}$ & $\mathrm{~S}_{1} \rightarrow \mathrm{S}_{0}$ & - \\
Cyclohexane & 0.98 & 276 & 339 & &
\end{tabular}

${ }^{a}$ Total lifetime of fluorescence. ${ }^{b}$ Wavelength of the maximum of the absorption spectrum. ${ }^{c}$ Wavelength of the maximum of the fluorescence spectrum. ${ }^{d}$ Fluorescence quantum yield.
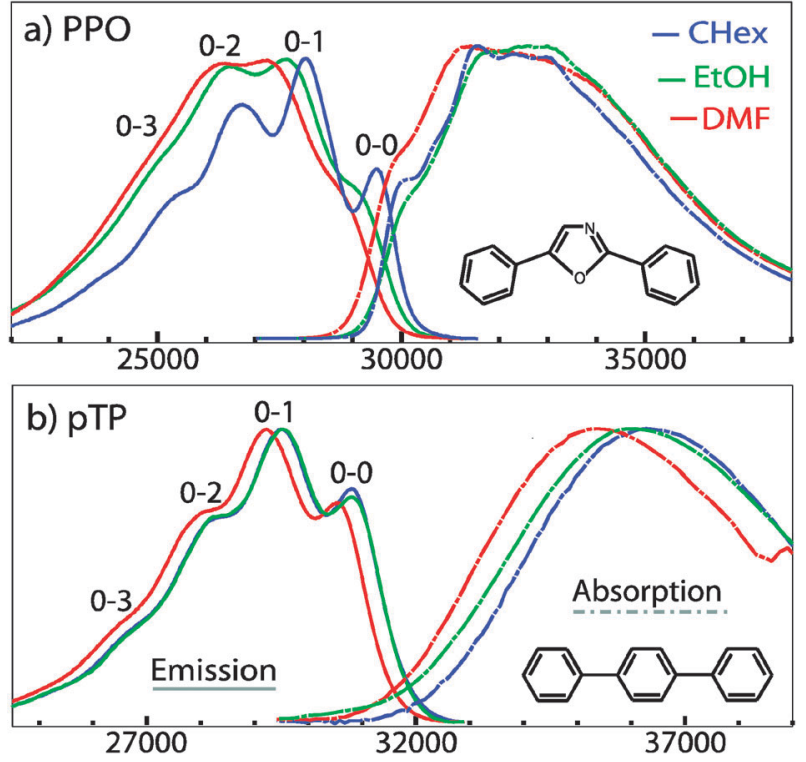

Fig. 1 Steady-state absorption (discontinuous line) and emission (continuous line) spectra of (a) 2,5-diphenyloxazole (PPO), (b) paraterphenyl ( $p \mathrm{TP})$ in cyclohexane (CHex, blue), ethanol (EtOH, green) and dimethylformamide (DMF, red). The structures of the dye molecules are shown in the inset.

in the field of $\pi$-conjugated materials for its prospective use in optoelectronic applications. ${ }^{37,38} p \mathrm{TP}$ is apolar and its steady state absorption and emission spectra do not show significant changes in the different solvents. Nevertheless, the mirror image symmetry rule is clearly violated, the absorption being broad and featureless, contrary to the structured emission. This reflects an important conformational change upon excitation. ${ }^{39}$ In the ground state, the weak single $\mathrm{C}-\mathrm{C}$ bonds connecting the phenyl rings imply the presence of low frequency librational modes. The related potential is characterized by an anharmonic double minimum well, with minima at an angle of $\sim 30^{\circ}$ with respect to the planar configuration. In the excited state, the phenyl-phenyl bonds are strengthened and the molecule becomes planar with a near harmonic potential. While the absorption is smeared out by the wide vibronic progression from the torsional motion, the emission spectrum is well-structured (Fig. 1), and is characterized by the coupling with two modes of frequency 1614 and $1286 \mathrm{~cm}^{-1}$, according to the fluorescence spectrum of crystalline $p$ TP at $4.2 \mathrm{~K}^{40,41}$

Since we are interested in the vibrational relaxation processes of these two dyes, excitation with a large excess energy is required. The $290 \mathrm{~nm}$ excitation used in the present experiment corresponds to an excess vibrational energy of $\sim 4500 \mathrm{~cm}^{-1}$ in PPO and $\sim 2500 \mathrm{~cm}^{-1}$ in $p \mathrm{TP}$, with respect to the absorption threshold.

\section{Materials and methods}

Our ultrafast broadband UV fluorescence set-up is based on the up-conversion scheme, and has been previously described..$^{5,20,42}$ Briefly, we use $290 \mathrm{~nm}$ pulses with a bandwidth of about $4 \mathrm{~nm}$ and a pulse width of about $70 \mathrm{fs}$ at a repetition rate of $250 \mathrm{kHz}$. The excitation energy of $30 \mathrm{~nJ}$ pulse $^{-1}$ was chosen to be in the linear regime of excitation. The UV-excited emission from the sample, circulated inside a 0.2 mm-thick quartz flow-cell, is filtered with a $310 \mathrm{~nm}$ cut-off filter to remove the remaining excitation light, and then mixed in a $250 \mu \mathrm{m}$-thick BBO crystal with the $800 \mathrm{~nm}$ gate beam. The up-converted signal is detected with a CCD camera-equipped monochromator. To overcome the phase-matching condition of the sum frequency crystal, the latter is continuously turned during the measurements. The use of the type I sum-frequency process imposes the detection polarization, which is orthogonal to the excitation. The temporal and spectral responses of $180 \mathrm{fs}$ and $6 \mathrm{~nm}$, respectively (fwhm), were determined by a measurement of the Raman response from the solvent (data not shown). The spectral correction was performed by normalization of the longest time spectrum to the steady state fluorescence spectrum, assuming that no spectral evolution occurs for times $>100$ ps.

Data analysis is performed in terms of spectral moments, with the first- $\left(M_{1}\right.$, average spectral position), and second-order $\left(M_{2}\right.$, spectral variance) moments being extracted from the data, as well as in terms of Franck-Condon fit of the transient spectra (see Section 3.2, Analysis). The vibrational normal modes of PPO and $p$ TP (frequencies reported in Table S1, ESI $\dagger$ ) were calculated using the Gaussian 09 program $^{43}$ at DFT level with the M06L $x$-c potential. ${ }^{44}$ A $6-31 \mathrm{~g}^{*}$ basis set was used.

\section{Results}

\section{Time-resolved spectra}

The normalized transient fluorescence spectra of PPO and $p$ TP in cyclohexane, ethanol and dimethylformamide, excited at $290 \mathrm{~nm}\left(34500 \mathrm{~cm}^{-1}\right)$, are shown in Fig. 2. While $70 \%$ of the non-normalized integrated intensity $\left(M_{0}(t)\right.$, Fig. S1, ESI $\left.\dagger\right)$ grow within our instrumental response function, the remaining $30 \%$ grow with a time constant of $\sim 60 \mathrm{ps}$, which due to our polarized detection, we associate to rotational diffusion of the dye, in agreement with previous studies. ${ }^{45,46}$ In all solvents, the 


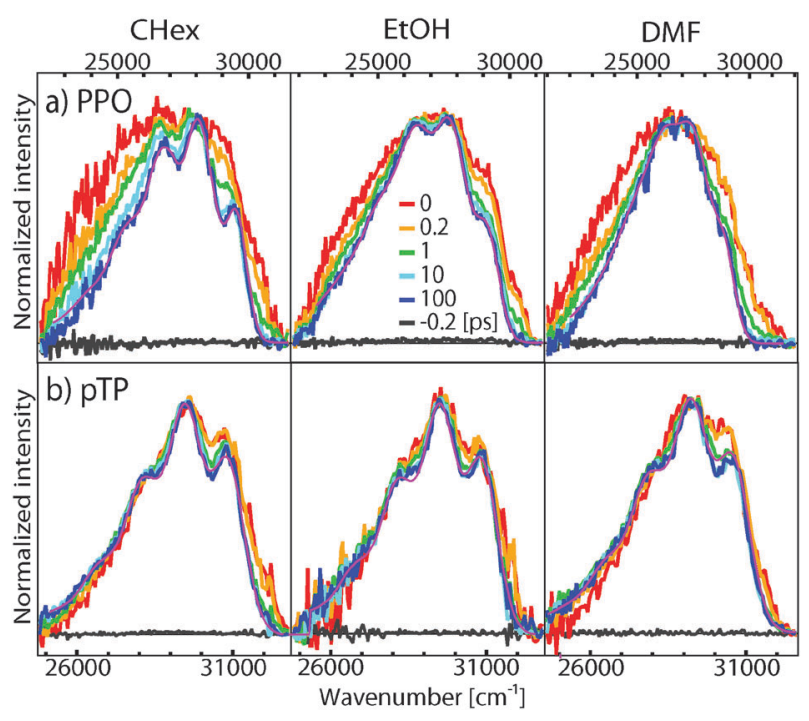

Fig. 2 Representative set of fluorescence spectra from (a) PPO and (b) $p$ TP in cyclohexane (left), ethanol (middle) and dimethylformamide (right) at different time delays, upon $290 \mathrm{~nm}$ excitation. The purple trace is the $\mathrm{F}-\mathrm{C}$ fit at $t=100 \mathrm{ps}$.

spectra at time zero are already centered close to the maximum of the steady state fluorescence band. Accordingly, most of the total Stokes shift $\left(\sim 5000 \mathrm{~cm}^{-1}\right)$ occur within our instrumental response. This promptly relaxed fluorescence band with one maximum speaks for emission stemming from a vibrationally cold level, as far as high frequency modes are concerned. A similar behaviour was recently reported for a large class of metal-based molecular complexes. ${ }^{47}$ The emission bands at early times are broad and featureless, contrary to those at long times. The blue-most part of the spectrum was cut by the filter at $310 \mathrm{~nm}$ (see above). From the end of the excitation pulse (orange trace, $t=200 \mathrm{fs}$ ) to the trace recorded at the longest time delay (dark blue, $t=100 \mathrm{ps}$ ), the spectrum narrows down progressively, converging to the steady state fluorescence before 100 ps. For PPO, this narrowing is most noticeable in cyclohexane where the spectrum at 100 ps dramatically differs from that at time zero.

The early time transient spectra of PPO show a peculiar evolution (red, orange and green traces) which is due to an oscillatory behaviour. This is clearly observed in the kinetic traces taken at the blue and red wings of the spectra (Fig. 3)

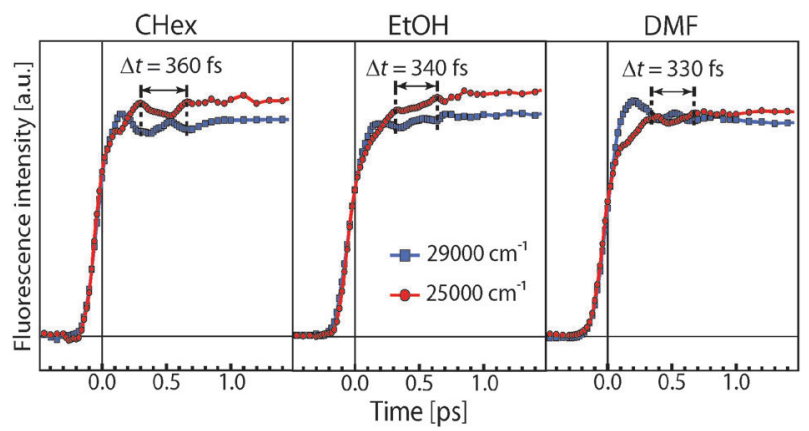

Fig. 3 Fluorescence kinetic traces at $350 \mathrm{~nm}\left(29000 \mathrm{~cm}^{-1}\right)$ and $400 \mathrm{~nm}\left(25000 \mathrm{~cm}^{-1}\right)$ of PPO in cyclohexane (left), ethanol (middle) and dimethylformamide (right), from the data shown in Fig. 2. where a damped oscillation with a period of 330-360 fs can be seen. The opposition of phase between the red and the blue wings suggests an oscillation of the position of the entire band, due to wavepacket dynamics between the turning points of the excited state potential on which it evolves.

In the case of $p$ TP, no such behaviour is observed. The intensity on the blue edge decreases as a function of time, whereas that on the red edge increases. As will later be seen, this red shift of the entire band is most likely due to intramolecular relaxation, since the Franck-Condon maximum does not shift, ruling out any contribution from solvation dynamics.

\section{Analysis}

In order to reliably interpret the data, a spectral moment analysis (see Materials and methods) was applied and compared to a Franck-Condon $(\mathrm{F}-\mathrm{C})$ fit analysis. The transient spectra were in fact adjusted with a $\mathrm{F}-\mathrm{C}$ progression including the coupling with two high frequency modes: ${ }^{33,40}$

$$
I(\nu, t)=A(t) \frac{e^{-\left(S_{1}+S_{2}\right)}}{\sigma(t)} \sum_{n, m=0} \frac{S_{1}^{n} S_{2}^{m}}{n ! m !} e^{-\left(\frac{\nu-\nu_{0-0}(t)+n \nu_{1}+m \nu_{2}}{\sigma(t)}\right)^{2}}
$$

In this fit, only the amplitude $(A)$, the origin of the transition $\left(v_{0-0}\right)$ and the width $(\sigma)$ are adjustable parameters. All other parameters, given in Table 2, are set to the values found in the literature for the frequencies $\left(v_{1}, v_{2}\right)$ and to those obtained from the fit of the static spectrum for the Huang-Rhys factors $\left(S_{1}, S_{2}\right)$. The only assumption required to perform such an analysis is that the emission stems uniquely from one excited state, vibrationally cold with respect to the high frequency $\mathrm{F}-\mathrm{C}$ modes, i.e. that IVR has already occurred. The time-dependent contribution to the width describes the broadening resulting from the temperatureinduced population of the low frequency modes: ${ }^{48}$

$$
\sigma^{2}(T)=N S\langle v\rangle^{2} \operatorname{coth}\left(\frac{h\langle v\rangle}{2 k_{\mathrm{B}} T}\right)
$$

where $v$ is the average frequency of degeneracy $N$ and average linear coupling constant $S$. For temperature higher than the low average frequency $\left(k_{\mathrm{B}} T \gg h\langle\nu\rangle\right)$, the above relation becomes linear:

$$
\sigma^{2}(T) \sim \frac{N S\langle v\rangle 2 k_{\mathrm{B}} T}{h}
$$

The fits were satisfactory for all spectra (Fig. S2, ESI $\dagger$ ), except in the case of PPO for times $<1 \mathrm{ps.} \mathrm{The} \mathrm{time}$ dependence of the first spectral moment $M_{1}(t)$ and of the energy of the zero-phonon-line as obtained from the fit is shown in Fig. 4. As previously observed, the evolution of the position of the band is clearly different in PPO and $p$ TP. The early time evolution of PPO exhibits a damped oscillatory

Table 2 Parameters used for the F-C fit (eqn (1)). The Huang-Rhys factors $S_{1}$ and $S_{2}$ were obtained by the fit of the static spectrum

\begin{tabular}{lllll}
\hline Compound & $v_{1} / \mathrm{cm}^{-1}$ & $S_{1}$ & $v_{2} / \mathrm{cm}^{-1}$ & $S_{2}$ \\
\hline PPO & $1544^{33}$ & 1.21 & $980^{33}$ & 0.86 \\
$p$ TP & $1614^{40}$ & 0.42 & $1286^{40}$ & 0.93 \\
\hline
\end{tabular}




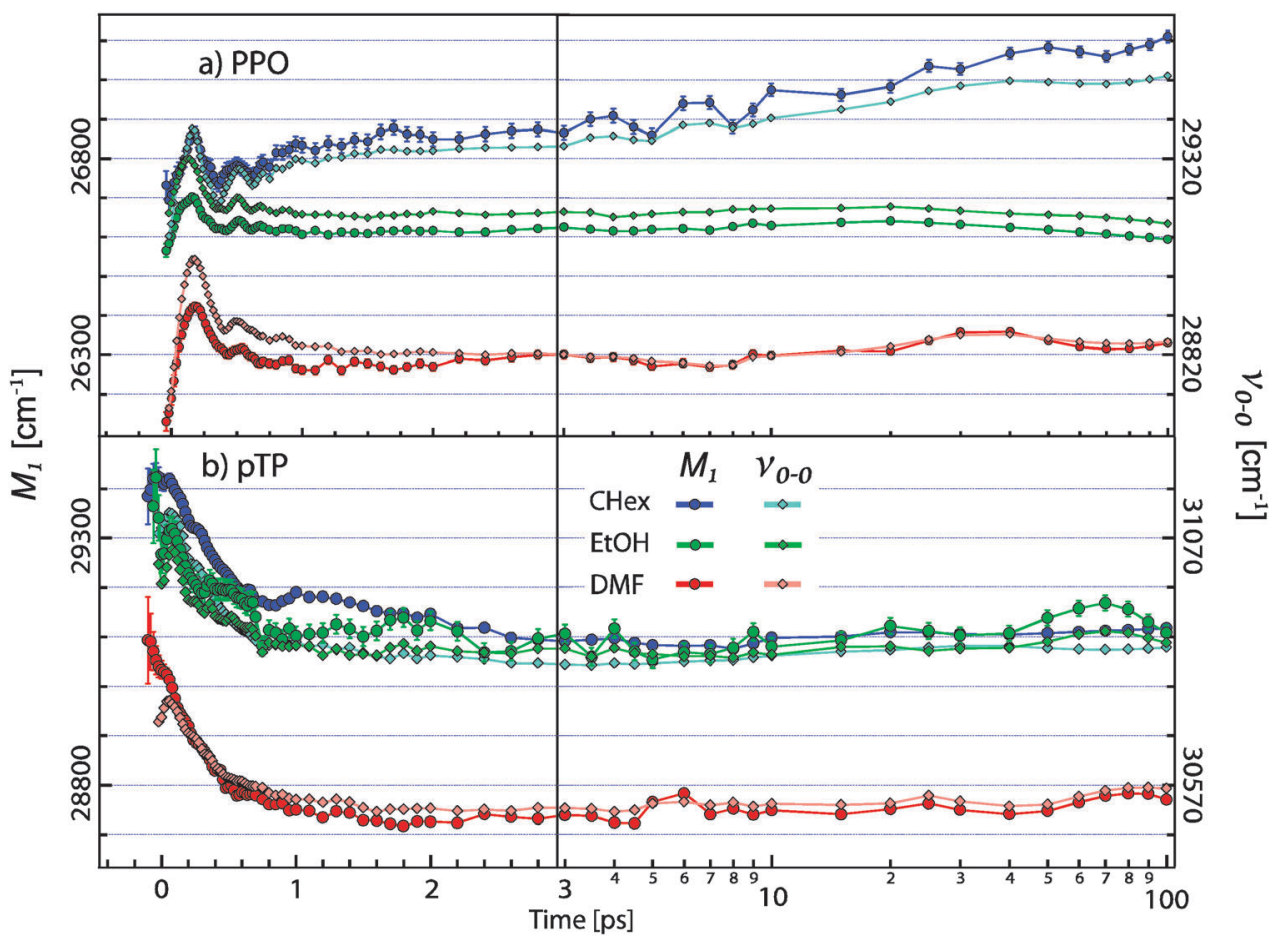

Fig. 4 Average fluorescence spectral position $M_{1}(t)$ (left scale) and zero-phonon-line (right scale) of (a) PPO and (b) $p$ TP in CHex, EtOH and DMF. The first 3 ps are in a linear scale, whereas from 3 to 100 ps the time is in a logarithmic scale.

pattern, due to a low frequency vibrational wavepacket. This wavepacket might be directly generated by the excitation, or indirectly through the ultrafast IVR. However, because of the important structural changes upon promotion to the excited state (as suggested by the breakdown of the mirror image symmetry in the static absorption/emission spectra in Fig. 1) and the low symmetry of PPO $\left(C_{\mathrm{s}}\right)$, all the vibrational modes are very likely $\mathrm{F}-\mathrm{C}$ active and a wavepacket is probably directly excited.

Its period of $\sim 350$ fs corresponds to a frequency of $\sim 95 \mathrm{~cm}^{-1}$. A Fourier analysis of the oscillatory pattern, shown in Fig. S3 (ESI $\dagger$ ), confirms a main contribution at $100 \pm 30 \mathrm{~cm}^{-1}$ for all the solvents $(\tau=330 \pm 90 \mathrm{fs})$. The damping constant is $300-500 \mathrm{fs}$, as estimated by the ratio between the first and second maximum in the oscillations of $M_{1}(t)$ (Fig. S3, ESI $\dagger$ ).

After $1 \mathrm{ps}$, the band has reached its steady-state position in ethanol and dimethylformamide, whereas a multimodal $300 \mathrm{~cm}^{-1}$ blue shift still occurs in cyclohexane. Concerning $p$ TP, a $\sim 300 \mathrm{~cm}^{-1}$ red shift of the band occurs in the first $\mathrm{ps}$, with similar amplitude and timescales in all solvents.

The band narrowing (Fig. 2) is reflected as a decay in the second moment $M_{2}(t)$ shown in Fig. 5, and in the width $\sigma^{2}$ of the fit (Fig. S2, ESI $\dagger$ ). The latter matches $M_{2}$ at every time delay when scaled by an appropriate factor, except in the region where the fit is not satisfactory, i.e. in the first ps (not shown). This spectral narrowing is typical of a cooling process. In order to establish the relationship between the width of the fluorescence and the temperature of the system, the static fluorescence spectra of both dyes were acquired for different temperatures ranging from 260 to $380 \mathrm{~K}$ in all solvents (see Fig. S4 (ESI $\dagger$ ) for cyclohexane). $\dagger$ The variance

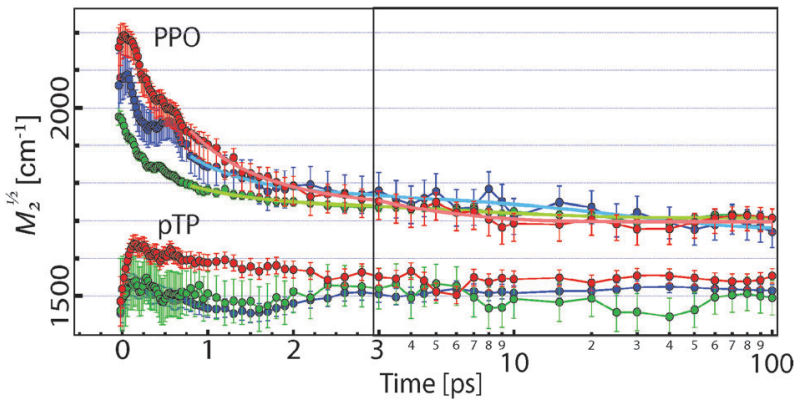

Fig. 5 Square root of the fluorescence spectral variance $M_{2}(t)^{\frac{1}{2}}$ of PPO and $p \mathrm{TP}$ in CHex (blue), EtOH (green) and DMF (red). The first 3 ps are in a linear scale, whereas from 3 to $100 \mathrm{ps}$ the time is in a logarithmic scale.

$\sigma^{2}$ appears linear with temperature, allowing us to extrapolate the temperature of the molecule at every time delay.

Fig. 6 shows the molecular temperature of PPO and $p$ TP for delay times where the fit is satisfactory. In DMF, the fluorescence spectrum of PPO is not structured enough to extract a bandwidth.

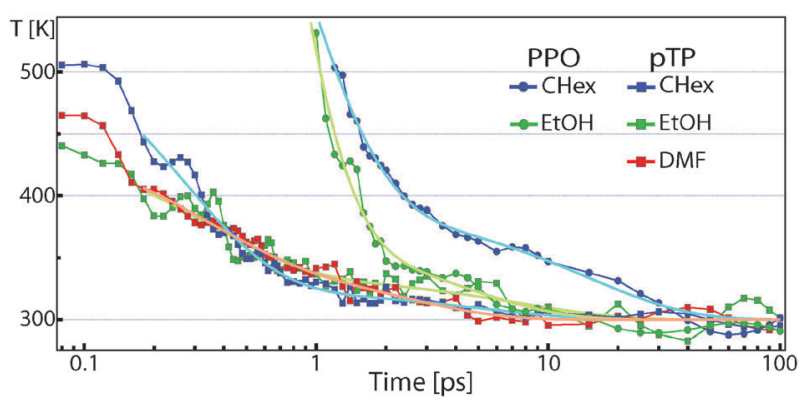

Fig. 6 Molecular temperature of $\mathrm{PPO}$ and $p \mathrm{TP}$ in $\mathrm{CHex}$, EtOH and DMF. 
The temperature of PPO and $p$ TP after IVR, i.e. when the population of each vibrational mode is described by a Boltzmann distribution, but before thermalization with the solvent has occurred, is related to the excess vibrational energy $\Delta E_{\mathrm{vib}}$ via: ${ }^{49}$

$$
E_{\mathrm{vib}}^{\mathrm{tot}}(T)=\sum_{i}^{3 N-6} \frac{h v_{i}}{e^{h v_{i} / k_{\mathrm{B}} T}-1}
$$

where $\nu_{i}$ is the frequency of the $i$ th mode and $E_{\mathrm{vib}}^{\mathrm{tot}}$ is the total vibrational energy $\left(E_{\mathrm{vib}}^{\mathrm{tot}}=E_{\mathrm{vib}}^{\mathrm{i}}+\Delta E_{\mathrm{vib}}, i=\right.$ initial, and $\Delta E_{\mathrm{vib}}=$ $\sim 4500 \mathrm{~cm}^{-1}$ and $\sim 2500 \mathrm{~cm}^{-1}$ for PPO and $p$ TP respectively). The vibrational frequencies of PPO and $p \mathrm{TP}$ (Table S1, ESI $\dagger$ ) were introduced in the above equation in order to extract the initial molecular temperature. The latter is found to be $\sim 510 \mathrm{~K}$ and $\sim 420 \mathrm{~K}$ for the estimated excess of energy in PPO and $p$ TP, respectively.

At $\sim 1$ ps after excitation, the temperature of PPO is found to be $\sim 500 \mathrm{~K}$, in agreement with the computed value. The spectra at earlier time delays cannot be satisfactorily fitted with the FranckCondon progression. Yet, the narrowing mainly occurs in the first ps, as shown by the decrease of $M_{2}(t)^{\frac{1}{2}}$ from $>2000$ to $1850 \mathrm{~cm}^{-1}$ (Fig. 5). However, such a value of $M_{2}^{\frac{1}{2}}$ at time zero would correspond to an initial temperature $>2000 \mathrm{~K}$, strongly deviating from the calculated temperature and physically meaningless. This is even more so that the early spectra are cut on the blue side and the calculated values of $M_{2}$ at early times are surely underestimated.

In the case of $p \mathrm{TP}$, the initial temperature extrapolated in Fig. 6 is $460 \mathrm{~K}$ for $\mathrm{EtOH}, 500 \mathrm{~K}$ for DMF and $520 \mathrm{~K}$ for $\mathrm{CHex}$, with an uncertainty of $\sim 40 \mathrm{~K}$. These values exceed the above estimated temperature of $420 \mathrm{~K}$. The intramolecular temperature is only reached after $\sim 200 \mathrm{fs}$, i.e. at the end of the excitation pulse. Table 3 reports the temperature amplitudes and decay times obtained from a bi-exponential fit performed from 200 fs to the last delay time.

These observations confirm that in the early transient regime ( $<$ ps for PPO and $<200$ fs for $p$ TP), the fluorescence cannot be described by the $\mathrm{F}-\mathrm{C}$ progression (eqn (1)). As will be discussed in the next section, in this regime structural changes might occur (accompanied by the launching of a coherent vibrational wavepacket in the case of PPO), which significantly affect the fluorescence spectrum.

\section{Discussion}

The F-C analysis of the time-resolved fluorescence spectra requires two hypotheses. First, the definition of the temperature is possible only when the population of each vibrational mode is given by a Boltzmann distribution, i.e. once IVR processes are over. Second, no important structural changes occur. We have evidence that one or both of these hypotheses does not hold in the sub-ps domain:

(1) As mentioned above, the spectra of PPO before $\sim 1 \mathrm{ps}$ cannot be satisfactorily fitted with the Franck-Condon progression of the static spectrum (eqn (1)).
(2) The normalized spectra in Fig. 2 show a change in the relative peak height of the $0-0,0-1$ and $0-2$ Franck-Condon bands, which is not described with the former treatment. This observation and the presence of wavepacket dynamics in the case of PPO speak for a non-thermalized system.

(3) The breakdown of the mirror image symmetry in the static absorption/emission spectra (Fig. 1) suggests important structural changes in the excited state. These changes, very likely involving out-of-plane distortion as demonstrated in the case of $p \mathrm{TP},{ }^{39}$ must occur on a given timescale ranging from the fs to the ps regime.

The latter remark is especially obvious in $p \mathrm{TP}$, and is due to an important structural change in the excited state. ${ }^{39}$ It is tempting to assume that the early dynamics observed in the first moment of $p \mathrm{TP}$ and $\mathrm{PPO}$ are triggered by such structural changes. In the case of PPO, the time zero spectrum resembles the mirror image of the absorption (broader than emission), suggesting that the structural change has still not occurred. The coordinate describing the conformational change might involve one (or a few) vibrational mode(s) slow enough to generate a coherent vibrational wavepacket. The latter dynamics would then modulate the fluorescence spectra in this transient regime.

The complete set of frequency modes calculated in the ground electronic state using Gaussian 09 are given in Table S1 and the 8 lowest ones are shown in Fig. S5 (ESI $\dagger$ ). Ionescu et al. ${ }^{28}$ reported the excited state optimized geometries of a derivative compound of PPO, showing an increase in the phenyl-oxazole bond order and a decrease in that of the $\mathrm{C}=\mathrm{N}$ and $\mathrm{C}=\mathrm{C}$ bonds inside the oxazole ring. These changes suggest an in-plane phenyl-oxazolephenyl bending contraction. The third calculated vibration of frequency $67 \mathrm{~cm}^{-1}$ corresponds to such a vibrational deformation (Fig. S5, ESI $\dagger$ ) and might be involved in the wavepacket. However, the next vibrational mode of $89 \mathrm{~cm}^{-1}$ is a combination of out-of-plane torsional and waving motion. This mode matches the observed oscillation frequency better and is most likely the main contribution to the wavepacket. The time dependent spectral shift probably originates from oscillation of the system at the bottom of the excited state potential well, along the coordinate of the involved vibrational mode. The energy stored in this mode is redistributed either to lower frequency modes, or directly to the solvent. Indeed, polar interaction of the closest solvent molecules with the oxygen and nitrogen atoms on the oxazole moiety would damp more efficiently the wavepacket dynamics. However, the uncertainty in the determination of the damping constant does not allow us to evidence a solvent dependence.

In the case of $p \mathrm{TP}$, no oscillation is observed, but the same considerations apply: the fluorescence maximum is at its final position already at time zero, whereas the zero-phonon-line decreases and narrows concomitantly with an increase of the red wing, resulting in the red shift of $M_{1}(t)$ (Fig. 4). As mentioned before, the structural rearrangement of $p \mathrm{TP}$ is associated to a planarization, mainly involving the torsional

Table 3 Time constants of a bi-exponential fit of $T(t)$ from Fig. 6 (amplitudes in parenthesis)

\begin{tabular}{lllll}
\hline Solvent & $\tau_{1}{ }^{\mathrm{PPO}} / \mathrm{fs}(T / \mathrm{K})$ & $\tau_{2}{ }^{\mathrm{PPO}} / \mathrm{ps}(T / \mathrm{K})$ & $\tau_{1}{ }^{p \mathrm{TP}} / \mathrm{fs}(T / \mathrm{K})$ & $\tau_{2}{ }^{{ }^{\mathrm{TP}}} / \mathrm{ps}(T / \mathrm{K})$ \\
\hline Cyclohexane & $700 \pm 60(125 \pm 10)$ & $20 \pm 3(75 \pm 7)$ & $270 \pm 30(90 \pm 10)$ & $4.2 \pm 1.2(30 \pm 5)$ \\
Ethanol & $400 \pm 50(130 \pm 10)$ & $5.6 \pm 1.6(70 \pm 7)$ & $300 \pm 50(80 \pm 10)$ & $7.6 \pm 2.8(40 \pm 5)$ \\
Dimethylformamide & - & - & $310 \pm 40(80 \pm 10)$ & $2.3 \pm 0.5(40 \pm 5)$ \\
\hline
\end{tabular}


motion of the phenyl rings. ${ }^{39}$ The effect of such a hot low frequency mode on the emission spectrum of $p$ TP corresponds to a strong broadening and a related blue shift of the band, consistent with the distortion of the early transient spectra of $p$ TP. Accordingly, the early dynamics of $p$ TP $(t<200$ fs in Fig. 4 and 6) are likely due to torsional relaxation towards the planar configuration.

In summary, the sub-ps dynamics of PPO and $p$ TP observed in the first moment reflect some structural changes induced by the photo-excitation. While their manifestations are different, i.e. damping of a wavepacket in PPO and red shift in $p$ TP, they are both signatures of similar processes, related to structural changes in the excited state. The faster relaxation process in $p \mathrm{TP}$ agrees with the fact that the $\mathrm{F}-\mathrm{C}$ progression still reasonably fits the early time spectra. This is not the case for PPO, which shows a time zero spectrum comparable to the mirror image of the absorption, i.e. that cannot be reasonably adjusted by the $\mathrm{F}-\mathrm{C}$ progression.

The following spectral changes, namely the band narrowing, correspond to the release of the excess of vibrational energy to the solvent, i.e. the cooling of the molecule. The latter dynamics is surprisingly fast. The main part of the cooling $(80-90 \mathrm{~K})$ occurs on a sub-ps timescale (200-300 fs, Table 3), whereas only $40 \mathrm{~K}$ thermalizes on the picosecond timescale. The fast decay is independent of the solvent for $p \mathrm{TP}$, whereas it is accelerated in the polar one for PPO. Within our uncertainty, we do not observe any effect of the solvent on the slowest decay times. Nevertheless, PPO in cyclohexane shows a blue shift in tens of ps (Fig. 4). This unusual behaviour is unclear, but it seems to be related to the solvent polarity. The emission/absorption mirror image symmetry being most marked in cyclohexane, it is tempting to relate this behaviour to some further structural rearrangements that are absent or hindered in polar solvents.

\section{Conclusions}

The set of data presented above allows us to describe the vibrational relaxation mechanisms of 2,5-diphenyloxazole and para-terphenyl excited with a large excess of vibrational energy (respectively 4500 and $2500 \mathrm{~cm}^{-1}$ ). The main part of intramolecular Stokes shift is almost instantaneous ( $<60 \mathrm{fs}$ ), meaning that the energy initially localized in the Franck-Condon high frequency modes $\left(1544(1614) \mathrm{cm}^{-1}, v=3(2)\right.$ for PPO $\left.(p T P)\right)$ is very rapidly redistributed by IVR to lower frequency modes.

In the case of PPO, the broad spectrum oscillates around its average equilibrium position within the first picosecond, with a period of $\sim 350 \mathrm{fs}$. The dephasing of this wavepacket accompanies the initial fast narrowing of the band. For $p$ TP, no coherent vibrations are observed, but a sub-ps red shift characterizes the first moment of the band. This feature, as for PPO, is the last step of IVR, and more specifically, a vibrational relaxation of the phenyls torsional motion. However, the contribution from IVR is less than in the case of PPO, since the $\mathrm{F}-\mathrm{C}$ fit is still satisfactory at time zero, and the estimated temperature is reached after only $\sim 200 \mathrm{fs}$. These results suggest a faster IVR in $p \mathrm{TP}$, which can be explained by the larger number of degrees of freedom in $p \mathrm{TP}$, the presence of heavier atoms $(\mathrm{N}, \mathrm{O})$ in the oxazole ring of $\mathrm{PPO}$, as well as the higher symmetry of $p$ TP compared to PPO.
The vibrational energy transfer to the solvent is bi-exponential for both dyes. Its sub-picosecond onset occurs on the same timescale as the vibrational relaxation process described above, while the complete thermalization is achieved in tens of ps. This biphasic vibrational cooling could tentatively be explained by a two-step energy transfer: in the first picosecond the chromophore transfers its energy to the closest solvent molecule (soluteto-solvent transfer), while the subsequent cooling is limited by transfer of the heat to the bulk (solvent-to-solvent transfer). This local heating effect, giving rise to the initial cooling dynamics, is enhanced by the polarity of the solvent, which strengthens the solute-solvent interaction. ${ }^{10}$ Accordingly, the initial cooling rate of $p \mathrm{TP}$ is independent of solvent polarity, as expected for an apolar solute. However, the fact that the initial cooling occurs on the same timescale as the damping of the wavepacket dynamics could support another explanation for the origin of the former: the vibrational cooling could tentatively depend on the distribution of population among all vibrational modes, making the process of cooling highly non-exponential. The cooling is faster in the beginning and progressively slows down, finally showing up as a bi-exponential decay.

We have thus been able to identify the presence of a subpicosecond solvent-induced vibrational relaxation, separated by the slower heat dissipation to the bulk. This study reveals the importance of considering the conformational changes in the fluorescence spectrum at short delay time after photoexcitation. Moreover, it illustrates the limits of the definition of the temperature of a molecule in the femtosecond domain, in particular when IVR processes interfere with cooling dynamics.

\section{Acknowledgements}

The authors would like to thank Dr Ahmad Oskouei Ajdarzadeh and Cristina Consani for helpful discussions.

\section{Notes and references}

1 S. Zalis, A. Cannizzo, A. M. Blanco-Rodriguez, A. El Nahhas, J. Sebera, A. Vlcek and M. Chergui, J. Am. Chem. Soc., 2008, 130, 8967-8974.

2 J. Briand, O. Braem, J. Rehault, J. Leonard, A. Cannizzo, M. Chergui, V. Zanirato, M. Olivucci, J. Helbing and S. Haacke, Phys. Chem. Chem. Phys., 2010, 12, 3178-3187.

3 R. J. Sension, S. T. Repinec, A. Z. Szarka and R. M. Hochstrasser, J. Chem. Phys., 1993, 98, 6291-6315.

4 G. Zgrablic, S. Haacke and M. Chergui, J. Phys. Chem. B, 2009, 113, 4384-4393.

5 G. Zgrablic, K. Voitchovsky, M. Kindermann, S. Haacke and M. Chergui, Biophys. J., 2005, 88, 2779-2788.

6 O. Braem, C. Consani, A. Cannizzo and M. Chergui, J. Phys. Chem. B, 2011, 115, 13723-13730.

7 C. Consani, O. Braem, F. van Mourik, A. Cannizzo and M. Chergui, Chem. Phys., 2011, DOI: 10.1016/ j.chemphys.2011.1009.1002.

8 J. Assmann, A. Charvat, D. Schwarzer, C. Kappel, K. Luther and B. Abel, J. Phys. Chem. A, 2002, 106, 5197-5201.

9 T. Elsaesser and W. Kaiser, Annu. Rev. Phys. Chem., 1991, 42, 83-107.

10 S. A. Kovalenko, R. Schanz, H. Hennig and N. P. Ernsting, J. Chem. Phys., 2001, 115, 3256-3273.

11 D. Schwarzer, J. Troe and M. Zerezke, J. Chem. Phys., 1997, 107, $8380-8390$.

12 O. Braem, A. A. Oskouei, A. Tortschanoff, F. van Mourik, M. Madrid, J. Echave, A. Cannizzo and M. Chergui, J. Phys. Chem. A, 2010, 114, 9034-9042. 
13 M. L. Horng, J. A. Gardecki, A. Papazyan and M. Maroncelli, J. Phys. Chem., 1995, 99, 17311-17337.

14 R. Jimenez, G. R. Fleming, P. V. Kumar and M. Maroncelli, Nature, 1994, 369, 471-473.

15 J. Kim, W. Y. Lu, W. H. Qiu, L. J. Wang, M. Caffrey and D. P. Zhong, J. Phys. Chem. B, 2006, 110, 21994-22000.

16 S. K. Pal, J. Peon and A. H. Zewail, Proc. Natl. Acad. Sci. U. S. A., 2002, 99, 1763-1768.

17 A. Mokhtari, A. Chebira and J. Chesnoy, J. Opt. Soc. Am. B, 1990, 7, 1551-1557.

18 T. Kiba, S. Sato, S. Akimoto, T. Kasajima and I. Yamazaki, J. Photochem. Photobiol., A, 2006, 178, 201-207.

19 A. Pigliucci, G. Duvanel, L. M. L. Daku and E. Vauthey, J. Phys. Chem. A, 2007, 111, 6135-6145.

20 A. Cannizzo, O. Braem, G. Zgrablic, A. Tortschanoff, A. A. Oskouei, F. van Mourik and M. Chergui, Opt. Lett., 2007, 32, 3555-3557.

21 D. Franco, G. Consolati and D. Trezzi, Phys. Rev. C: Nucl. Phys., $2011,83,015504$.

22 Y. Homma, Y. Murase and K. Sonehara, Int. J. Radiat. Appl. Instrum., Part A, 1987, 38, 91-96.

23 P. Ramasamy, N. Balamurugan and A. Arulchakkaravarthi, J. Cryst. Growth, 2008, 310, 2115-2119.

24 H. L. Xiao, X. B. Li, D. Zheng, J. Cao, L. J. Wen and N. Y. Wang, Chin. Phys. C, 2010, 34, 1724-1728.

25 C. Hariharan, V. Vijaysree and A. K. Mishra, J. Lumin., 1997, 75, 205-211.

26 B. Steyer and F. P. Schafer, Appl. Phys., 1975, 7, 113-122.

27 N. Boens, W. W. Qin, N. Basaric, J. Hofkens, M. Ameloot, J. Pouget, J. P. Lefevre, B. Valeur, E. Gratton, M. Vandeven, N. D. Silva, Y. Engelborghs, K. Willaert, A. Sillen, G. Rumbles, D. Phillips, A. J. W. G. Visser, A. van Hoek, J. R. Lakowicz, H. Malak, I. Gryczynski, A. G. Szabo, D. T. Krajcarski, N. Tamai and A. Miura, Anal. Chem., 2007, 79, 2137-2149.

28 S. Ionescu, D. Popovici, A. T. Balaban and M. Hillebrand, Spectrochim. Acta, Part A, 2005, 62, 252-260.

29 S. Ionescu, D. Popovici, A. T. Balaban and M. Hillebrand, Spectrochim. Acta, Part A, 2007, 66, 1165-1170.

30 I. B. Berlman, Handbook of Fluorescence Spectra of Aromatic Molecules, Academic Press, 1971.

31 A. A. Elazhary, A. A. Ghoneim and M. E. Elshakre, J. Chem. Res., Synop., 1995, 9, 354-355.

32 A. E. Obukhov, Zh. Fiz. Khim., 1995, 69, 1015-1024.

33 E. A. Mangle, P. R. Salvi, R. J. Babbit, A. L. Motyka and M. R. Topp, Chem. Phys. Lett., 1987, 133, 214-221.
34 W. P. Ambrose and W. E. Moerner, Nature, 1991, 349, 225-227.

35 W. E. Moerner and D. P. Fromm, Rev. Sci. Instrum., 2003, 74, $3597-3619$.

36 W. E. Moerner and M. Orrit, Science, 1999, 283, 1670-1676.

37 M. T. Bernius, M. Inbasekaran, J. O'Brien and W. S. Wu, $A d v$. Mater., 2000, 12, 1737-1750.

38 M. Gross, D. C. Muller, H. G. Nothofer, U. Scherf, D. Neher, C. Brauchle and K. Meerholz, Nature, 2000, 405, 661-665.

39 G. Heimel, M. Daghofer, J. Gierschner, E. J. W. List, A. C. Grimsdale, K. Mullen, D. Beljonne, J. L. Bredas and E. Zojer, J. Chem. Phys., 2005, 122, 054501.

40 C. Kryschi, W. Klufer and H. Kupka, Chem. Phys., 1990, 146, 231-236.

41 C. Kryschi, W. Klufer and H. Kupka, J. Lumin., 1991, 48(9), 381-384.

42 D. S. Larsen, M. Vengris, M. A. van der Horst, G. Zgrablic, I. H. M. van Stokkum, S. Haacke, M. Chergui, K. J. Hellingwerf and R. van Grondelle, Biophys. J., 2004, 87, 1848-1857.

43 M. J. Frisch, G. W. Trucks, H. B. Schlegel, G. E. Scuseria, M. A. Robb, J. R. Cheeseman, G. Scalmani, V. Barone, B. Mennucci, G. A. Petersson, H. Nakatsuji, M. Caricato, X. Li, H. P. Hratchian, A. F. Izmaylov, J. Bloino, G. Zheng, J. L. Sonnenberg, M. Hada, M. Ehara, K. Toyota, R. Fukuda, J. Hasegawa, M. Ishida, T. Nakajima, Y. Honda, O. Kitao, H. Nakai, T. Vreven, J. A. Montgomery, J. E. Peralta, F. Ogliaro, M. Bearpark, J. J. Heyd, E. Brothers, K. N. Kudin, V. N. Staroverov, R. Kobayashi, J. Normand, K. Raghavachari, A. Rendell, J. C. Burant, S. S. Iyengar, J. Tomasi, M. Cossi, N. Rega, J. M. Millam, M. Klene, J. E. Knox, J. B. Cross, V. Bakken, C. Adamo, J. Jaramillo, R. Gomperts, R. E. Stratmann, O. Yazyev, A. J. Austin, R. Cammi, C. Pomelli, J. W. Ochterski, R. L. Martin, K. Morokuma, V. G. Zakrzewski, G. A. Voth, P. Salvador, J. J. Dannenberg, S. Dapprich, A. D. Daniels, O. Farkas, J. B. Foresman, J. V. Ortiz, J. Cioslowski and D. J. Fox, Gaussian 09, Revision A.02, ed. G. Inc, Wallingford CT, 2009.

44 Y. Zhao and D. G. Truhlar, J. Chem. Phys., 2006, 125, 194101.

45 R. Luchowski, Chem. Phys. Lett., 2011, 501, 572-574.

46 J. R. Lakowicz, I. Gryczynski, Z. Gryczynski, E. Danielsen and M. J. Wirth, J. Phys. Chem., 1992, 96, 3000-3006.

47 O. Braem, F. Messina, A. M. El-Zohry, A. Cannizzo and M. Chergui, Chem. Phys., 2011, 393, 51-57.

48 J. J. Markham, Rev. Mod. Phys., 1959, 31, 956-989.

49 E. R. Henry, W. A. Eaton and R. M. Hochstrasser, Proc. Natl. Acad. Sci. U. S. A., 1986, 83, 8982-8986. 